current, similar to an inactivating current $I_{\mathrm{A}}$ described in molluscan neurones; a calcium current, $I_{\mathrm{Ca}}$; and a calciumdependent potassium current, $I_{\mathrm{K}(\mathrm{Ca})}$. These currents are known from other preparations, but a novel feature of the $I_{\mathrm{Ca}}$ described is that it has a fast activation $(<5 \mathrm{~ms})$ and seems not to inactivate. The sensitivity of the membrane oscillations and $I_{\mathrm{K}(\mathrm{Ca})}$ to TEA $^{10}$ suggests that at least one of the currents underlying this type of tuning may have been pinned down. A similar pharmacological dissection technique has been used to uncover ionic mechanisms in photoreceptors, where, at the last count, seven distinct currents have been described ${ }^{17}$. It would be surprising if the count in hair cells does not increase from three.

It remains to be shown that modelling the interplay of these currents can account quantitatively for tuning in saccular hair cells, where resonant frequencies extend to about $160 \mathrm{~Hz}$ (ref.18). Harder to answer is whether the same results apply to other hair cells where frequency-tuning occurs over a greater range. Does regulation of the internal calcium levels or the amplification produced by involving an internal messenger ion generate a wider range of possible frequencies? Most tantalizing is the developmental question: can this type of cellular mechanism be regulated to produce tonotopic organization in an auditory papilla? In contrast, perhaps von Békésy was describing the simplest engineering principle on which to build a cochlea.

Jonathan Ashmore is in the Department of Physiology, The Medical School, Bristol BS\& ITD.

\title{
Neurobiology
}

\section{Transfer of information in the visual cortex}

from Nicholas $V$. Swindale

THE paper by Livingstone and Hubel in Nature this week ${ }^{1}$ provides a further example of the importance of cytochrome oxidase patches, first discovered by Margaret Wong-Riley in the upper layers of the primary visual cortex of primates, in disentangling the anatomical relationships between the columns of neurones that represent the functional subdivisions of the visual cortex. The importance of the patches lies in their use as anatomical markers, since they have a fixed spatial relationship with at least one and probably several other types of column, while the histological method used to demonstrate them is simple and can easily be used in combination with other methods for studying columnar structure.

There are several possible explanations for the existence of the patches. It can be assumed that increased levels of the enzyme are in the first place the result of an increased level of metabolic activity, and that this must be due either to chronically higher levels of activity in the neurones within the patches, or to the type or rate of transmitter synthesis that is going on ${ }^{2}$.

Two observations suggest that a higher level of activity is at least part of the explanation. First, the absolute levels of the enzyme within each patch are not greater than they are within layer IV, which stains heavily and uniformly. It has been shown that the patches receive a direct input from the lateral geniculate nucleus ${ }^{3}$, as does layer IV, and if activity in these terminals is the cause of the high levels of the enzyme in layer IV, it might also cause the elevated levels within the patches. Second, physiological studies show that cells in the patches lack orientation selectivity ${ }^{4}$, suggesting that they would probably respond to a greater variety of stimuli than other cortical neurones. Many also have little or no low-spatial-frequency cut-off ${ }^{5}$, implying that they should respond to diffuse light, something that other cortical cells will not do.

Anatomically, the patches seem to be related to several different types of column. It is known that each patch is aligned with the centre of an ocular dominance stripe $^{6}$, and that each contains a high proportion of cells that respond selectively to coloured stimuli ${ }^{7}$. Thus it is likely that the patches either coincide, or intersect with the columns of colour-selective cells described by Michael $^{8}$. Recent work by Tootell and his colleagues ${ }^{9}$ suggests that the patches may relate to other types of column as well. Deoxyglucose studies where sine wave gratings of all orientations, but only one spatial frequency, are used to stimulate the cortex suggest that spatial frequency columns are arranged concentrically around the patches, cells between the patches being tuned to higher spatial frequencies than cells within the patches. Horton's and Hubel's original observation $^{6}$ that the patches intersect, or lie on the borders of orientation columns has been repeated by Tootell et al. ${ }^{9}$, strengthening the argument that different orientation columns must coalesce in or near the patches. The overall arrangement of columns that these results suggest, with orientation columns radiating out of the patches ranged concentrically around them, makes a good deal of sense, since it would allow every combination of spatial frequency and orientation to be represented within the diameter of an ocular dominance stripe. This is an important requirement if representation of visual information by the visual cortex is to be complete.

Livingstone and Hubel $^{1}$ have now taken the analysis one step further by using the cytochrome oxidase stain in conjunction with the horseradish peroxidase tracing method to study the organization of the projection from the primary visual cortex (area 17) to the neighbouring area 18. Cytochrome oxidase staining in area 18 reveals a pattern not of patches, but of short darkly staining strips, alternately thick and thin, and separated by narrow lightly staining strips ${ }^{10}$. The repeat periodicity (from one thin dark strip to the next) is about $2.5 \mathrm{~mm}$, probably the largest repeat period for any columnar structure yet discovered in the cerebral cortex (suggesting that other still lower periodicities might exist in other cortical areas). Livingstone and Hubel have now shown that the patches in area 17 project specifically to the thin dark strips in area 18 (the existence of a projection to the thick dark strips is equivocal), while conversely, non-patch regions in area 17 project to the palely staining inter-strip regions.

Two of Livingstone's and Hubel's findings are worth commenting upon. First, the region of cortex in area 17 that projects to an area 18 strip is about $2-3 \mathrm{~mm}$ wide substantially larger than the injected area within the strip, which is about $0.5 \mathrm{~mm}$ wide. Second, both left and right eye patches (those superimposed on left or right eye ocular dominance columns) participate in the projection. Thus there is an overall convergence of information - from both eyes, and from a relatively large region of area 17 - to single small regions in the area 18 strips. It is reasonable to assume that a columnar microstructure (with properties varying over distances of tens to hundreds of micrometres) exists within area 18 as it does in area 17. Just what properties might change on such a scale within the strips is not known, but they are presumably ones not possessed by cells within the area 17 patches. These two types of transformation, condensation of some features of the representation within area 17 , in parallel with the elaboration and creation of new ones, may turn out to be a general feature of the way in which information is transformed in the projection from one cerebral cortical area to another.

Nicholas $V$. Swindale is at the Department of Psychology, Dalhousie University, Halifax, Nova Scotia B3H $4 J 1$.

1. Livingstone, M.S. \& Hubel, D.H. Nature 304, 531 (1983)

2. Hendrichson, A.E., Hunt, S.P. \& Wu, J.W. Nature 292. 605 (1981).

3. Livingstone, M.S. \& Hubel, D.H. Proc, natn. Acad. Sci U.S.A. 79, 6098 (1982)

4. Hubel, D.H. \& Livingstone, M.S. Soc. Neurosci. Abstr. 7, 357 (1981).

5. Parker, A.J. \& Hawken, M.J. Invest. Opthalmol. Suppl. 22, 11 (1982).

6. Horton, J.C. \& Hubel, D.H. Nature 292, 762 (1981)

7. Hubel, D.H. \& Livingstone, M.S. Soc. Neurosci. Abstr. 8. 706 (1982).

8. Michael, C.R. J. Neurophysiol, 46, 587 (1981)

9. Tootell, R.B.H., Silverman, M.S., Switkes, E.\& De Valois, R.L. Soc. Neurosci. Abstr. 8, 707 (1982).

10. Tootell, R.B.H., Silverman, M.S., De Valolis, R.L. \& Jacobs, G.H. Science 220, 737 (1983). 